\title{
Traducir el blanco de las letras: semánticas de la memoria y sus evidencias cuali-cuantitativas
}

\section{Translating the White Side of Letters: Memory Semantics and their Quali-Quantitative Evidence}

\section{Rossella MICHIENZI}

Università della Calabria

michienzirossella@gmail.com

Recibido: julio 2018. Revisado: septiembre 2018. Aceptado: octubre 2018.

Resumen: Profundizando la relación entre Translation y Memory Studies e inspirándose en los conceptos de ideología y poder propuestos por Foucault (1975), Lefevere (1992) y Carbonell (1999), el artículo problematiza la representabilidad de eventos traumáticos a través del lenguaje. Para ello se considera el caso de los desaparecidos argentinos, intentando demonstrar por lo menos dos cosas: 1. La traducción representa un espacio fundamental para la negociación de significados, memorias u olvidos; 2 . el olvido puede evitarse sólo escuchando el ruido producido por los traumas. El trabajo se centra en la heteroglosia de términos que durante la última dictadura han ampliado sus connotaciones. Finalmente, a través del análisis de datos empíricos extraídos con el software de concordancias AntConc 3.5.3, se proporcionan evidencias cuantitativas de dichas mutaciones, ilustrando la frecuencia de uso de esos términos en el Nunca Más (1984).

Palabras clave: traducción; metamorfosis; worldmaking; análisis terminológico.

Rossella MICHIENZI

Traducir el blanco de las letras: semánticas de la memoria y sus evidencias cuali-cuantitativas 


\begin{abstract}
Going through the relation between Translation and Memory Studies, and inspired by the notions of ideology and power as proposed by Foucault (1975), Lefevere (1992) and Carbonell (1999), this contribution aims to set out a theoretical and empirical digression regarding representability of traumatic eventsthrough language (considering it as something that is never neutral). To this end, the case of the enforced disappearances in Argentina is studied, trying to demonstrate at least two things: 1. Translation represents a fundamental space for the negotiation of meanings, memories or oblivions; 2. oblivion can be avoided only by listening to the noises produced by traumatic events. This article focuses on the heteroglossia of these terms that widened their connotations during the last military dictatorship. Finally, through the analysis of empirical data extracted with the concordance software AntConc 3.5.3, this study provides quantitative evidences of such mutations, illustrating the frequency of use of these terms in the CONADEP commission's full report, Nunca Más (1984).
\end{abstract}

Key words: translation; metamorphosis; worldmaking; terminological analysis.

\title{
1. ¿TRADUCCIÓN O METAMORFOSIS?
}

En el espacio de la comunicación intercultural, la traducción está relacionada cada vez más con la circulación de culturas y conocimientos. Considerar el proceso de transposición interlingüística como instrumento que permite la difusión de informaciones significa llevar nuevamente esa práctica a su razón histórica primordial, ampliamente relacionada con el éxodo de los judíos a otras tierras, con la consiguiente necesidad de traducir a la segunda lengua adquirida el texto bíblico que los migrantes ya no podían leer en la lengua original. Por lo tanto, la traducción siempre implica una especie de drama, de la pérdida (pérdida de un sentido de pertenencia, pérdida de la lengua materna, pérdida de identidad) y traducir significa, de alguna forma, recuperar o preservar identidades creando ese "connecting thread between cultures, out of which the source culture has come to be linguistically and socially dependent in the globalization's era» (Babič 2018, 158). Sin embargo, sería reductivo pensar en la traducción en términos de simple acción comunicativa. La traducción sufre un peligroso empobrecimiento cuando se la considera un mero método instrumental para la reproducción de la fijeza semántica en las lenguas, las culturas y las mentalidades encerradas tras su propia sensación de autosuficiencia (Vidal Claramonte, 2017). Por eso, especialmente en la contemporaneidad, teoría y práctica de la traducción tienen que oponer a una matriz de carácter funcionalista una poética traductiva, una teoría del ritmo, entendida no simplemente en términos de atención a elementos fono-prosódicos del original, sino también a otros factores, entre los cuales se incluye también la actitud del sujeto con respecto a cuanto (re)formula y (re)vive en traducción.

Algunos enfoques teóricos establecen que los traductores deberían evitar que sus propias creencias y valores intervengan en el proceso traductivo y que los matices ideológicos presentes en el original deberían transmitirse sin verse afectados por el 
punto de vista del mundo que tiene quien traduce. Se trata de una visión que da lugar a una increíble simplificación de la figura del traductor y del proceso de interpretación que él mismo cumple. La traducción es, más bien, un proceso de reproducción que lleva a la creación de un texto que tiene vida propia y que no se puede considerar simple copia del original. Siendo el traductor el reflejo de las estructuras sociales en que vive, es imposible que intervenga en el proceso de traducción alejándose de su propia realidad o evitando lo que Peter Hanenberg, desde una perspectiva psico-cognitivista, ha definido como intramental translation (Hanenberg 2018), refiriéndose a ese proceso con que se traduce una información en un significado y en el cual lo que el sujeto percibe, a través de la experiencia, coincide con sus modelos culturales.

Es evidente que cada vez que el traductor elige una palabra, una estructura, un esquema rítmico y no otro participa activamente en la creación de conocimiento, moldeando culturas:

[Translation] is not simply an act of faithful reproduction but, rather, a deliberate and conscious act of selection, assemblage, structuration, and fabrication [...] translators, as much as creative writers and politicians, participate in the powerful acts that create knowledge and shape culture (Tymoczko y Gentzler 2002, xxi).

Aceptando la idea según la cual los modelos culturales de cada uno influencian el acto traductivo y que «ideology rather than linguistics or aesthetics crucially determines the operational choices of translation» (Cronin 2000, 695), se vuelve necesario reflexionar sobre la responsabilidad ética del traductor, precisamente porque, a través del lenguaje, es posible describir, construir o modificar la realidad, poner en discusión el sentido común, fomentar o extirpar estereotipos, atribuir identidades, pero también quitarlas. De ello, se deduce que no sólo el complejo oficio de la traducción está destinado a convertirse en acción indispensable para una real sostenibilidad (inter)cultural, sino que el proceso de metamorfosis que implica la traducción es uno de los espacios más importantes para la negociación de significados y de relaciones de poder entre culturas y memorias diferentes.

Como el lenguaje, la traducción es una práctica que se produce en estrecha relación con un contexto y unos vínculos de carácter ideológico. Cada sujeto, cuando habla, coloniza las palabras con sus propias intenciones y connotaciones tanto semánticas como expresivas, realizando un proceso de apropiación de la lengua. Pero, antes de ese proceso, la lengua no existe y no puede existir, en una forma neutral o impersonal; existe más bien en las intenciones o connotaciones de los demás (Bachtin 1992). Es ahí que cada uno de nosotros debería tomar la palabra y hacerla propia. Es lo que ocurre en traducción, ya que, como sugiere Roland Barthes (1983), no tenemos relaciones neutras con los signos; por lo tanto, el trabajo del traductor no es, ni puede ser, invisible o imperceptible en el universo heteroglósico del cual se compone cualquier texto. 
Paul Ricoeur considera el acto traductivo no simplemente como un desafío, sino como un sufrimiento debido a la indiscutible diferencia entre el "yo" y el "otro" que implica la necesidad de abandonar el sueño de una traducción perfecta. Acercar el lector al autor o, al revés, el autor al lector (aun a riesgo de servir y/o traicionar a dos amos) significa practicar lo que el filósofo francés llama «hospitalidad lingüística» (Ricoeur 2001, 49) y coincide con la capacidad del traductor de adentrarse en un viaje hacia la alteridad. Sólo entregándose a la diferencia, yendo más allá de las palabras, escarbando en sus significados más íntimos, el traductor puede habitar la lengua del "otro» y, al mismo tiempo, experimentar la felicidad de recibir en su propia casa la palabra del extranjero. Es evidente que cuando se habla de traducción es imposible huir de su sentido metafórico que abarca la idea del traslado, del viaje, del éxodo, del desplazamiento y necesariamente de la transformación de un texto, al pasar de una cultura de origen a una cultura de llegada.

En un reciente estudio, Jonathan P.A. Sell (2018), a partir de un famoso verso de la comedia de Shakespeare, A Midsummer Night Dream («Bless, thee, Bottom, bless thee. Thou art translated» $[3.2,112])^{1}$ ha subrayado la fuerte relación existente entre el concepto de traducción y su acepción griega de metamorfosis, estimulando la reflexión sobre los problemas que surgen en traducción cuando «the host semiosphere refuses to recognize the identity of a text which does not comply with the linguistic, conceptual and aesthetic scripts or stereotypes in terms of which it constructs its own [...] identities» (Sell 2018, 52).

Por lo tanto, a partir de las reflexiones de Sell y volviendo a las ideas de Lionel Trilling (1971) sobre el concepto de autenticidad del texto literario, es posible afirmar que cuando la cultura de llegada (host semiosfere) privilegia su propia identidad, perjudicando la identidad del texto original, el resultado es una traducción inauténtica, casi ilegítima, que ha padecido una incuestionable coerción. Sell intenta ir más allá del principal dilema de la traducción literaria (domesticación vs. extranjerización). Precisamente, ya que traducir, de alguna manera, significa domesticar y la única extranjerización total consistiría en no traducir, y ya que el traductor realiza ambas operaciones inconscientemente, al mismo tiempo y en diferentes grados, se halla una posible solución en la «metamorphic translation» (Sell 2018, 29), o sea, en una traducción que consiga ser metamórfica, «recognizable as both its original self and as something else» (Sell 2018, 53), como Bottom: un ser trasformado cuya identidad, aun así, sigue siendo reconocible. Una traducción metamórfica mantiene suficientes elementos para que el texto sea reconocible en sus rasgos originales y es adaptada suficientemente para que pueda sobrevivir en la cultura de llegada.

1. La famosa exclamación de Peter Quince al ver a Nick Bottom cuya cabeza humana había sido cambiada por la de un asno.

Rossella MICHIENZI

Traducir el blanco de las letras: semánticas de la memoria y sus evidencias cuali-cuantitativas 
En otras palabras, un proceso de traducción metamórfica lleva a la creación de un texto ajeno a sí mismo - «a stranger to itself» como diría Kristeva (1991)- que sigue manteniendo su propia identidad. El resultado podría considerarse como algo fragmentario o carente, pero como insinúa Sell:

[...] a metamorphic translation, like a human subject, will and should never be complete or perfect, and thank goodness for that: it is that very incompleteness and imperfection that makes recognition possible (Sell 2018, 49).

Dicho proceso traductológico conlleva inevitables dualismos, y la consiguiente hibridación, el aspecto incompleto e imperfecto del texto son, exactamente, aquellos elementos que permiten el reconocimiento y la tutela de espacios y tiempos culturales diferentes. Además, como sugiere Berman (2003), la fuerza de una lengua no consiste en su capacidad de rechazar la diversidad, sino en su destreza en devorarla; la capacidad de devorar al otro se traduce en términos de curiosidad hacia el otro y de negación del vacío que vive entre nosotros y lo que nos es diferente. En ese vacío se realiza la pérdida semántica que caracteriza cada acto traductológico, y es ahí donde se desarrolla, simultáneamente, dentro de los límites de lo decible y de la afasia.

Parecería que sólo la metamorfosis (verwandlung, como escribe Kafka en su obra maestra), considerada como constante traducción de una experiencia vital a otras experiencias vitales, puede permitir una relación ética con el mundo. La metamorfosis en traducción representa esa capacidad de abandonar una visión etnocéntrica para emprender una nuevo y sorprendente viaje hacia la heteroglosia que atraviesa las culturas.

\section{SOBRE LA POSIBILIDAD DE UNA HERMENÉUTICA DEL SILENCIO ENTRE MEMORIA Y OLVIDO}

El encuentro con la alteridad supone un encuentro con culturas diferentes, pero también con diferentes historias y memorias. Como la traducción, la memoria tiene sus aristas peligrosas: puede alterar eventos, transmutar realidades y ser utilizada por diferentes grupos para sus propios intereses (Todorov 1995). La destreza metamórfica de la memoria se realiza a través de representaciones performativas. Por eso, es imposible ignorar la relación circular e interdependiente que existe entre lenguas y memorias. Cabe destacar que la memoria no tiene que ver con el pasado; ella es, más bien, el presente del pasado y señala la posibilidad de activar este último en el presente de cada ser humano, lo que define la identidad personal y la continuidad del sí mismo en el tiempo. Los recuerdos individuales no se producen de manera personal, sino que se ubican dentro de cadres sociaux que les dan sentido (Halbwachs 1987). Por consiguiente, toda memoria, aun la más privada, es constitutivamente social y, 
obviamente, plural. Además, si «la interrogación sobre el pasado es siempre activa» (Aletta de Sylvas 2011, 143), este último, construido en diálogo e interacción, deja de ser algo inmutable. Por eso, la memoria no siempre es fiel a la verdad.

Como la memoria, también el trauma deriva de una atribución de significados socialmente mediada por narrativas sociales y códigos simbólicos, de tal modo que la comunidad traumatizada genera una nueva narrativa maestra de sufrimiento social. Por ejemplo, el proceso representativo a través del cual la Shoah ha llegado a ser reconocida como símbolo del mal absoluto tiene que ver con las narrativas usadas para codificarla en el discurso público americano. Como escribe Jeffrey Alexander en su ensayo «On the Social Construction of Moral Universals»:

[...] no trauma interprets itself; before trauma can be experienced at the collective level, there are essential questions that must be answered, and answers to those questions change over time. For a traumatic event to have the status of evil is a matter of its becoming evil. It is a matter of how the trauma is known, how it is coded (Alexander 2004, 202).

En otras palabras, «becoming evil is a matter, first and foremost, of representation» (Alexander 2004, 203). Construimos la realidad a través del lenguaje que usamos, por lo tanto, en la construcción cultural de los traumas, la codificación lingüística desempeña un papel fundamental, permitiendo que las cosas se instalen o se erradiquen dentro de la esfera pública, plasmando realidades y creando mundos posibles.

Los discursos producidos a raíz del trauma argentino son ejemplares para una reflexión sobre el poder de las palabras:

[...] cada palabra siempre evoca, siempre trae consigo ruidos [...] cada palabra le llega al traductor construida a base de cicatrices, cargada de historias, repleta de ideologías, moldeada previamente por otras manos de colores, sexos y religiones diferentes (Vidal Claramonte 2017, 42).

Los ruidos que "cada palabra trae consigo" nos obligan a reflexionar sobre la carga política del lenguaje y, por lo tanto, de la traducción. Traducir implica entender que «what counts is no longer what a text says, but what it does. Its power, no longer merely meaning» (Meschonnic 2011, 85) y que lo más peligroso del lenguaje (y de la traducción) es precisamente lo que dice sin decir. No es el significado denotativo de las palabras, sino su carga connotativa lo que se tiene que «escuchar» (Vidal Claramonte 2017) de un texto, a la hora de traducirlo.

Si los eventos traumáticos producen ruidos y éstos son los elementos más complicados de entender, el objetivo principal del traductor sería «[...] to translate not what is there but what is not there, [...] the blank spaces between the words" (Bielsa y Bassnett 2009, 6). Hay que imaginar cada palabra como si fuese un enorme iceberg

Rossella MICHIENZI

Traducir el blanco de las letras: semánticas de la memoria y sus evidencias cuali-cuantitativas 
cuya parte supuestamente más peligrosa queda oculta a la vista. De ahí, surge la necesidad de practicar una hermenéutica del silencio, siendo siempre conscientes de que ése no existe; lo que se manifiesta en cuanto tal es lo implícito escondido en los espacios blancos que yacen, supuestamente inertes y sin vida entre las líneas oscuras de las palabras.

\subsection{El caso argentino y la ecuación de la palabra-iceberg}

La última dictadura argentina fue un régimen pluridimensional que, con su metodología de terror, llevó a la desaparición de miles de seres humanos, dañando la sociedad a varios niveles. Es muy sugestiva la imagen que representa la multiplicidad de los efectos del terror como un potente chorro de agua que cae sobre una roca (Samojedny 1986): el chorro no hace más que mojar la roca, pero el agua, cayendo repetidamente, gota tras gota, en el mismo punto, acaba por desgastarla. El trauma argentino ha deteriorado la estabilidad emotiva de los familiares de las víctimas, las condiciones físicas de los detenidos y su sistema psíquico mediante las torturas, las relaciones interpersonales de individuos forzados al aislamiento y, finalmente, los sentimientos (todas las expresiones emotivas, como reír o llorar, en los CCD² estaban prohibidas). A todo esto, hay que añadir el clima de terror que se difundía entre la gente. Miedo y terror fueron factores determinantes del comportamiento social y político, tanto a nivel individual como a nivel colectivo. La complejidad del cuadro impide que se hable de traumas individuales, se trata, más bien, de un acontecimiento que produjo muchos traumas individuales y que, al mismo tiempo, pertenece a la colectividad.

Tal como los individuos y las colectividades, también el lenguaje, formando parte del orden social en el cual se realiza el evento, sufre una implosión. En este caso, el trauma de la lengua ha llevado a un proceso metamórfico que se ha concluido con la construcción de una «lengua del trauma» (Michienzi 2016) constituida por palabras violadas, estropeadas, transfiguradas. En Argentina, en 1976, empieza a construirse un nuevo sistema de significados vehiculados por términos ya existentes, que han ido ampliando sus connotaciones. Para decirlo de otra forma, la lengua se ha enriquecido gracias a nuevos significados (más o menos visibles y más o menos audibles) que se manifiestan a través del uso de viejos significantes. Generalmente mutaciones de este tipo son el resultado de una colonización, por eso el caso argentino provoca un innegable desconcierto ya que víctimas y verdugos pertenecen a un mismo mundo lingüístico. En esta lengua, común a los perpetradores y a las víctimas, se acuñó la palabra desaparecido en su acepción actual para referirse a alguien que ha sido privado

\section{Centros Clandestinos de Detención.}

Rossella MICHIENZI

Traducir el blanco de las letras: semánticas de la memoria y sus evidencias cuali-cuantitativas 
ilegalmente de su libertad (según el DRAE: «muerto», eufemismo usado también como sustantivo). Siempre en esta lengua, surgen, por ejemplo, la nueva acepción de asador (palabra que deja de referirse a "quien prepara el asado" para identificar a los «torturadores en los campos de detención y tortura») o de trasladar (palabra que deja de referirse sólo a la «acción de llevar a alguien o algo de un lugar a otro», para convertirse en sinónimo de «asesinar»). El análisis de estos significados escondidos ha hecho patentes las marcas -más o menos repudiadas- que la aniquilación ha dejado en la vida cotidiana de los argentinos.

Entrevistas realizadas en Argentina entre 2013 y 2015 y el análisis del trauma writing nacional (Michienzi 2015) han permitido aislar unos términos que se pueden definir Trauma Bound Terms (en adelante TBT), términos traumo-específicos, que han ido agregando a sus significados comunes, significados relativos a la experiencia traumática.

A continuación, a modo de ejemplo, se mencionan algunos de los términos-iceberg que aparecen en el Nunca Más (1984)3. Cada término está acompañado por la definición general (en adelante GD) que de ellos se encuentra en los diccionarios comunes de la lengua española y la acepción relacionada con el evento traumático (en adelante TBD). A continuación, se presentará un estudio cuantitativo de los términos-iceberg que aparecen en el Informe.

- Candado: GD 1. s.m. Cerradura suelta contenida en una caja de metal, que por medio de armellas asegura puertas, ventanas, tapas de cofres, maletas, etc.; 2. Hecharse / ponerse un candado a la boca loc. v. colloq. Callar o guardar un secreto. TBD 1. s.m Carcelero, guardia.

- Cantar: GD 1. v. intr. 1. Producir con la voz sonidos melodiosos; 2. v. tr. Interpretar con la voz una composición musical. TBD 1. v. tr/intr. Decir la verdad, confesar algo, generalmente bajo tortura (y no simplemente bajo presión); denunciar a los cómplices.

- Capucha: GD 1. s.f. Pieza de una prenda de vestir que sirve para cubrir la cabeza y se puede echar a la espalda. TBD 1. s.f. Bolsa con que se cubría la cabeza a los secuestrados para aislarlos 2. Nombre del sótano del Casino de Oficiales de la ESMA (Escuela de Mecánica de la Armada) que, entre 1976 y 1983, ha sido un CCD.

- Chupar: GD 1. v. tr. Sacar o traer con los labios y la lengua el jugo o la sustancia de algo 2. jerg. Cuba. Dicho de la policía: detener (Il prender). TBD 1. v. tr.

3. Informe oficial de la Comisión Nacional sobre la desaparición de personas que reúne miles de testimonios de las victimas (ex-desaparecidos) o parientes de las víctimas y que permite constatar como en Argentina, en aquellos años, los derechos humanos hayan sido bárbaramente violados.

Rossella MICHIENZI

Traducir el blanco de las letras: semánticas de la memoria y sus evidencias cuali-cuantitativas
CLINA

vol. 4-2, December 2018, 207-222

elSSN: 2444-1961

Ediciones Universidad de Salamanca - CC BY-NC-ND 
Detener ilegalmente o secuestrar a una persona; 2. Secuestrar a los «subversivos» para después detenerlos en CCD.

- Cortar: GD 1. v. tr. Dividir algo o separar sus partes con algún instrumento cortante. TBD 1. v. tr. Matar, quitar la vida a un ser vivo.

- Cura: GD 1. s.m. En la Iglesia católica, sacerdote encargado, en virtud del oficio que tiene, del cuidado, instrucción y doctrina espiritual de una feligresía; 2. m. coloq. Sacerdote católico. TBD 1. s.m. Militar de los Grupos de Tareas (GT), cuya función era la de interrogar a los detenidos para que «dieran informaciones» y "confesaran» sus acciones subversivas.

- Máquina [darse máquina / dar máquina a alguien]: GD 1. Artificio para aprovechar, dirigir o regular la acción de una fuerza; 2. Cuba, Coche TBD 1. Arg. Instrumento de tortura 2. (dar máquina) Torturar con picana eléctrica.

- Picana [p. eléctrica]: GD 1. s.f. Aguijada. Vara larga que en un extremo tiene una punta de hierro con que los boyeros pican a la yunta; 2. s.f. Instrumento que transmite electricidad. TBD 1. s.f. Instrumento de tortura con el que se aplican descargas eléctricas en cualquier parte del cuerpo de la víctima.

- Picanear: GD 1. v. tr. Aguijar a los bueyes. TBD 1. v. tr. (de picana) Torturar con picana eléctrica.

- Sala [de la felicidad]: GD 1. s.f. En un edificio público, habitación de grandes dimensiones. TBD 1. s.f. En algunos CCD, habitación en la cual los detenidos sufrían una tipología atípica de tortura psicológica: los verdugos les obligaban a escuchar siempre la misma canción o melodía (también durante las torturas físicas).

- Tabique: GD 1. s.m. Pared delgada que sirve para separar las piezas de la casa; 2. s.m. División plana y delgada que separa dos huecos. El tabique de las fosas nasales. TBD 1. Semejante a Capucha, técnica de tortura psicológica cuyos objetivos eran el aislamiento total de las víctimas y su total soledad y vulnerabilidad.

\subsubsection{Datos Empíricos}

Pese a que en esta fase de la investigación el corpus (entrevistas y relatos presentes en el Nunca Más) no pretende ser considerado exhaustivo, con sus 171394 palabras puede considerarse representativo. Como glosario de base se han utilizado los lemas señalados por Mario Sartor en La jerga de la Represión Argentina (1986), por Perla Sneh en Palabras para decirlo y otros términos que aparecen con acepciones diferentes a las comunes en las entrevistas (v. supra 2.1). Cabe destacar que, en el glosario, en su última versión, aparecen neologismos y términos cuya acepción traumoespecífica no aparece en los comunes diccionarios de la lengua española; se excluyen nombres propios de Centros Clandestinos de Detención (algunos de los cuales, por el 
contrario, aparecen en los glosarios que se acaban de mencionar) y otros términos que se consideran más bien como pertenecientes a la jerga militar. A continuación, la lista de los 78 términos presentes en el glosario que se ha usado como filtro para el trabajo:

\begin{tabular}{|c|c|c|c|}
\hline $\begin{array}{l}\text { - } \text { apagón } \\
\text { - } \text { apropiación } \\
\text { - } \text { apropiador, -dora } \\
\text { - } \text { asado } \\
\text { - } \text { asador } \\
\text { - } \text { atentado } \\
\text { - } \text { blanco } \\
\text { - } \text { blanco [en] } \\
\text { - } \text { boleta } \\
\text { - } \text { boletear } \\
\text { - } \text { botín [de g.] }]^{4} \\
\text { - cables } \\
\text { - candado } \\
\text { - } \text { cantar } \\
\text { - capacha } \\
\text { - capilla } \\
\text { - } \text { capucha } \\
\text { - casco [de la m.] } \\
\text { - chupado, -da } \\
\text { - chupadero }\end{array}$ & $\begin{array}{l}\text { - } \text { chupador, -ra } \\
\text { - } \text { chupar } \\
\text { - } \text { cortar } \\
\text { - } \text { cura } \\
\text { - } \text { desaparecer } \\
\text { - } \text { desaparecido, -da } \\
\text { - } \text { desaparición } \\
\text { - elemento [s. }]^{6} \\
\text { - } \text { enene } \\
\text { - enfrentamiento } \\
\text { - fusilado, -da } \\
\text { - golpe } \\
\text { - inmobiliaria } \\
\text { - laboratorio } \\
\text { - lavado de cerebro } \\
\text { - leonera } \\
\text { - máquina } \\
\text { - margarita } \\
\text { - moishe }\end{array}$ & 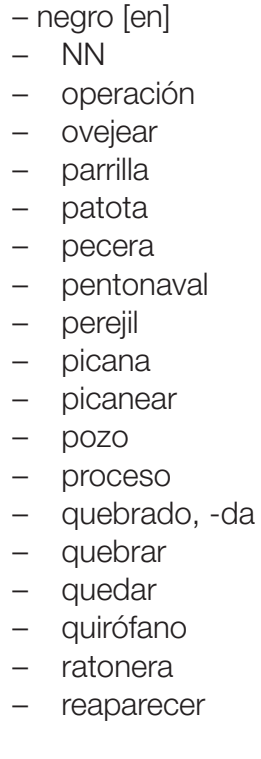 & $\begin{array}{ll}\text { - } & \text { reaparecido, -da } \\
\text { - } & \text { rectoscopio } \\
\text { - } & \text { represor, -ra } \\
\text { - } & \text { reprimido, -da } \\
- & \text { sala [de la f.] } \\
- & \text { sala [de t. i.] } \\
- & \text { sesión } \\
- & \text { submarino } \\
- & \text { subversivo, -va } \\
- & \text { subversivo, -va } \\
& \text { [e.] } \\
- & \text { sucia [guerra] } \\
- & \text { tabicado, -da } \\
- & \text { tabicar } \\
- & \text { tabique } \\
- & \text { teléfono } \\
- & \text { terror } \\
- & \text { trasladar } \\
- & \text { traslado } \\
- & \text { vuelo [de la m.] }{ }^{9}\end{array}$ \\
\hline
\end{tabular}

La identificación de los TBT ha sido realizada en tres fases diferentes: lectura y análisis del corpus, análisis de las unidades terminológicas extraídas con el programa de concordancias AntConc 3.5.3, y validación del listado de lemas relevado y la sucesiva desambiguación léxica a través de su relativo contexto lingüístico.

El número de TBT registrado suma 118 lemas, correspondientes a 1150 ocurrencias. Restringiendo el análisis. Se trata de 65 unidades léxicas de diferentes categorías gramaticales (sustantivos, adjetivos, verbos, participios y gerundios) que aparecen con sus diferentes declinaciones:

\footnotetext{
4. Botín de guerra.

5. Casco de la muerte (instrumento de tortura).

6. Elemento subversivo.

7. Sala de la felicidad (sala de tortura psicológica).

8. Sala de terapia intensiva (sala de tortura física).

9. Vuelo de la muerte (práctica de exterminio).
}

Rossella MICHIENZI

Traducir el blanco de las letras: semánticas de la memoria y sus evidencias cuali-cuantitativas 


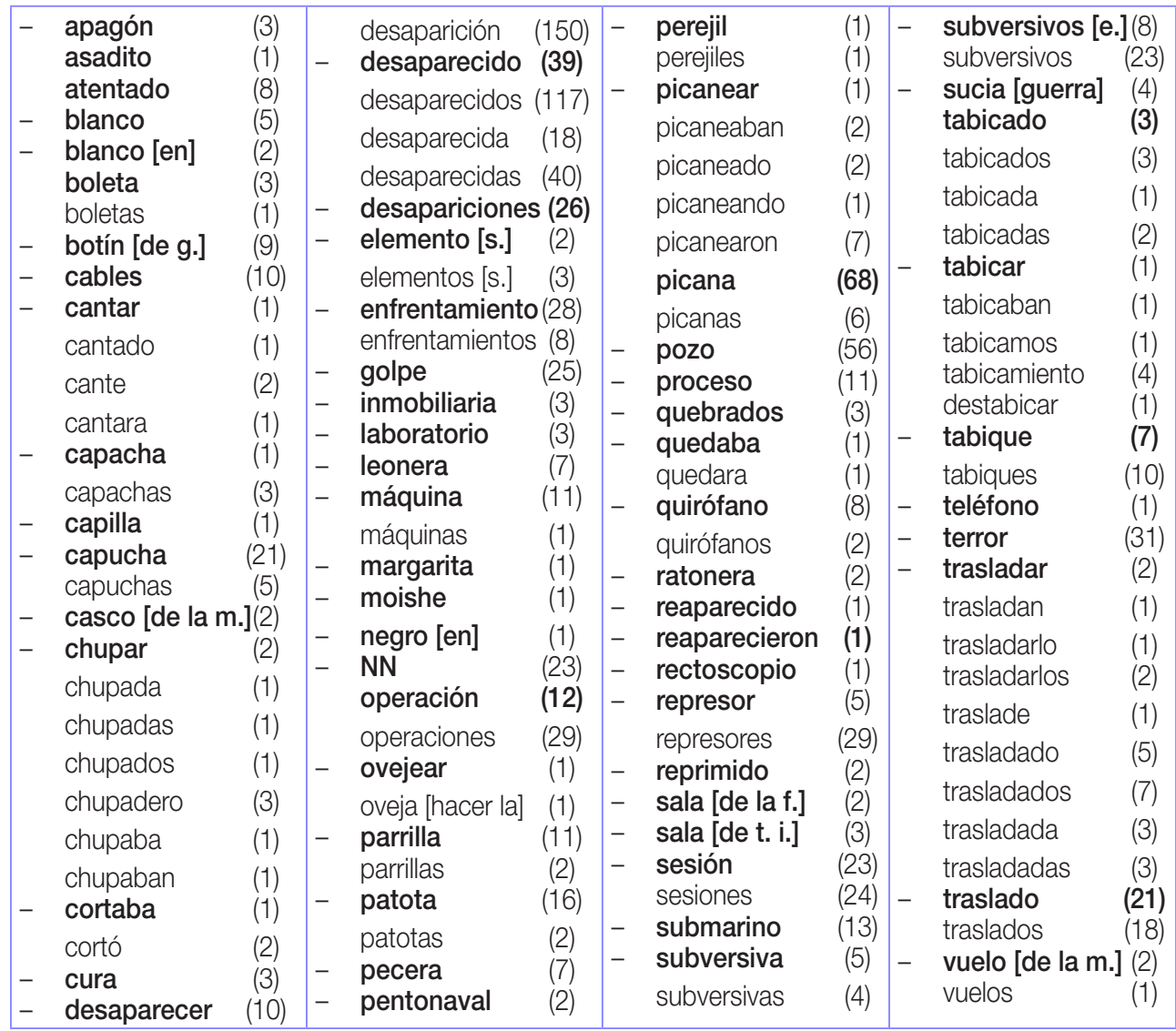

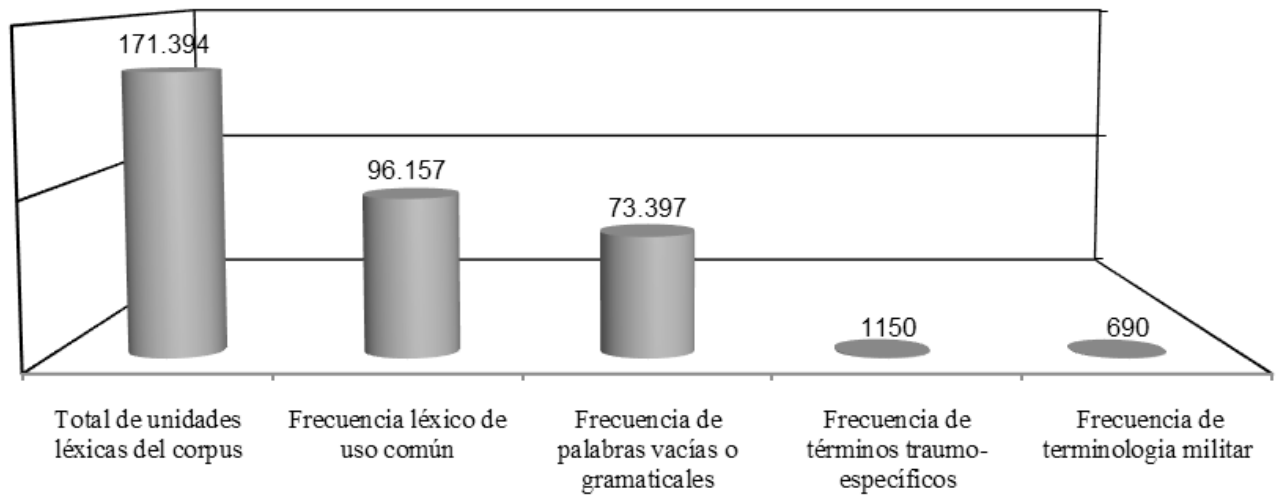

Figura 1. Índice de frecuencias

Rossella MICHIENZI Traducir el blanco de las letras: semánticas de la memoria y sus evidencias cuali-cuantitativas 
La Figura 1. representa la frecuencia de las unidades léxicas en el corpus. La primera columna de la izquierda corresponde al total de unidades léxicas del contenido documental, con sus 171.394 unidades. Dicho valor comprende tanto léxico y expresiones del lenguaje común como léxico y expresiones propias del lenguaje militar y términos que han cambiado su connotación. La segunda columna representa la frecuencia de términos de uso común (96 157 unidades); sigue la frecuencia de las 83 unidades léxicas que Anguiano Peña y C. Naumis Peña (2015) definen palabras vacías o gramaticales (artículos, preposiciones, interjecciones, pronombres, etc.) cuya frecuencia suma 73397 unidades (tercera columna). Sucesivamente se ha podido extraer el material léxico restante, constituido por 1840 unidades de las cuales 1150 (cuarta columna) equivalen a la frecuencia de los TBT y las restantes 690 (quinta columna) a la frecuencia de expresiones y siglas propias del lenguaje militar (GT, Lugar transitorio de detención, SIA, etc.).

El dato interesante es que el número de palabras usadas con acepción traumoespecifica es superior al número de términos pertenecientes a la jerga militar. Además, el proceso de desambiguación léxica ha permito aislar datos indicativos sobre el porcentaje de frecuencia de algunos términos con su doble acepción (común y traumoespecífica). Por ejemplo, el verbo trasladar, el sustantivo traslado y sus respectivos derivados aparecen en el corpus con acepción traumo-específica con un porcentaje de frecuencia del $22 \%$ respecto al total de las ocurrencias:

Dichos términos, cuya frecuencia suma 287 unidades (100\%), aparecen en el corpus con la acepción de «muerte», "asesinato», «ser asesinado» con una frecuencia de 64 unidades (22\%) y con la común acepción de «acción o efecto de trasladar» (DRAE) en el $78 \%$ de los casos (Figura 2.). El término máquina, con sus derivados, presenta en el corpus 22 unidades, 10 de las cuales remiten al significado común de «artificio para aprovechar, dirigir o regular la acción de una fuerza» (DRAE), las otras 12 aparecen como sinónimo de picana eléctrica, indicando, por consiguiente, el conocido instrumento de tortura. Destaca también el caso de tabique y del correspondiente verbo tabicar (v. supra 2.1) cuya frecuencia suma 46 unidades, de las cuales solo 12 aparecen con el significado común de «pared delgada que sirve para separar las piezas de la casa» (DRAE) y las restantes 34 unidades con acepción traumo-específica remitiendo a esa técnica de tortura psicológica, muy parecida a la de la capucha (bolsa con que se cubría la cabeza a los secuestrados), cuyos objetivos eran el aislamiento total de las víctimas y su total soledad y vulnerabilidad.

\section{PARA UNA SOSTENIBILIDAD (INTER)CULTURAL A TRAVÉS DEL LENGUAJE}

El estudio presentado, más allá del análisis cuali-cuantitativo, quiere remarcar los desafíos que implica la traducción de palabras-iceberg a lenguas, culturas y memorias

Rossella MICHIENZI

Traducir el blanco de las letras: semánticas de la memoria y sus evidencias cuali-cuantitativas
CLINA

vol. 4-2, December 2018, 207-222

elSSN: 2444-1961

Ediciones Universidad de Salamanca - CC BY-NC-ND 
diferentes. Es indudable que el lenguaje (y con él la traducción) crea discursos en torno a distintas identidades y a sus historias. En otras palabras, el lenguaje que elegimos al expresarnos y, entonces, al traducir, puede renovar memorias o, por el contrario, ejercer censuras, creando espacios oscuros que delimitan la nada. Sin embargo, como escribe en Cambio de Armas Luisa Valenzuela, las paredes de los pozos negros de la memoria «a veces resuenan» (Valenzuela 2016, 132) y las voces más profundas de lo que queda afuera de los confines del lenguaje aúllan de rabia dentro de los límites de lo que se podría enunciar y lo que se quiere aislar en la peligrosa vorágine del olvido.

Identidades, memorias y culturas, en el proceso hermenéutico que supone cualquier acto de transposición interlingüística e intercultural, se colocan en un espacio de constante traducción en el cual las relaciones de representación (y de conceptualización) se (re)escriben y (re)definen constantemente. Como se lee en La cultura como praxis de Zygmunt Bauman:

No hay punto de observación supracultural y suprahistórico (luego, libre de toda contingencia), desde el cual se pueda otear y retratar subsecuentemente el significado verdadero y universal [...]. La traducción es un proceso continuo, un diálogo inacabado e inconcluyente, destinado a permanecer así. El encuentro de dos contingencias es una contingencia en sí mismo y ningún esfuerzo hará que deje de serlo. El acto de la traducción nunca es un evento singular que hace innecesario cualquier otro intento de la misma naturaleza. El terreno de reunión, la tierra de frontera, de las culturas es el territorio en el cual se trazan continuamente unos límites sólo para violarlos y a volver a trazarlos una y otra vez [...]. La traducción transcultural es un proceso continuo que sirve a la cohabitación tanto como la constituye, de gentes que no se pueden permitir ocupar el mismo espacio ni cartografiar ese espacio común, cada uno a su manera (Bauman 2002, 85-86).

Por lo tanto, no hay acto de traducción que pueda dejar incólumes a las partes implicadas, las cuales salen de ese encuentro siempre diferentes. Traducir significa, necesariamente, manipular. En este sentido, es útil volver al concepto de transculturación, así como lo formula la crítica latinoamericana: un proceso mediante el cual el encuentro con la alteridad produce transformaciones en las sociedades puestas en contacto y elabora una respuesta resistente, una fuerza creativa en la transitividad entre culturas, aun cuando éstas se encuentran en diferentes posiciones de poder (Sales Salvador 2001). Se deduce que la transculturación, lejos de llevar a la desaparición de las distintas tradiciones culturales, implica su continuo desarrollo dentro de interacciones dialógicas. De alguna forma, es lo que supone la transposición metamórfica de Sell: si, por un lado, en traducción, algunos rasgos se pierden, por otro lado, otras peculiaridades se pueden ganar, generando nuevas potencialidades comunicativas y preservando la caída de conceptos, de historias y, sobre todo, de identidades dentro de los mencionados pozos negros de la memoria (Valenzuela 2016).

Rossella MICHIENZI

Traducir el blanco de las letras: semánticas de la memoria y sus evidencias cuali-cuantitativas 
Luchar contra representaciones dominantes, contra la creación y/o imposición de estereotipos, contra la aniquilación de identidades, es una tarea inevitable en los contextos sociales heterogéneos de una era global en la cual convergen y se entrelazan diferentes polisistemas. La metamorfosis, en traducción, implica la imposibilidad de evitar alteraciones, pero, al mismo tiempo, permite la coexistencia de diferentes realidades; dicha coexistencia, a su vez, lleva a la posibilidad de rescatar del olvido y del aislamiento discursos, historias y saberes que es necesario compartir y mantener vivos, desde la perspectiva del otro en cuanto tal.

Como sugiere Alejandro Gándara en Las primeras palabras de la creación, cada palabra es un mundo vivido que protege huellas y existencias previas y cuenta historias que, aunque se escondan, no se pueden ocultar:

Las palabras se ven y se leen sólo mediante distorsión. [...] Leemos con lágrimas de emoción y salimos de la lectura con las manos manchadas, no intactos, no aupados por encima del mundo, ni soplando como pequeños dioses sobre él. Salimos más cargados de mundo que antes, es más: con el mundo en las manos (Gándara 1998: 33).

Resulta innegable la importancia de subrayar la fuerte relación, interdependiente y circular, existente entre lenguas y memorias, a sabiendas de que la decodificación y la traducción de cada palabra supone hacerse cargo de cómo esa misma palabra ha llegado a ser lo que, efectivamente, es. Por lo tanto -como recuerda Ángel Gabilondo en un ensayo publicado en Siete sentencias sobre el séptimo ángel de Michel Foucault (1999)- la ecuación que supone cada palabra no tiene que ver simplemente con su arqueología o genealogía, ni con la comprensión de su etimología, sino con «las huellas y las marcas de otras vidas que confirman la suya» (cit. en Vidal Claramonte 2017, 142).

Desde una perspectiva terminológica, cuando una unidad lingüística tiene un significado comúnmente reconocido y otro latente que puede manifestarse en cualquier momento para evocar las cicatrices más profundas, (re)conocer la carga connotativa de la parte escondida del iceberg, significa atribuir una imagen al espacio, aparentemente sin forma, que se entrevé detrás de las palabras. En ese (re)conocimiento se esconde la posibilidad de defender horizontes ajenos, traducir el blanco de las letras y dejar que la lengua desempeñe su función primordial: permitir la comunicación y la circulación global de informaciones y de conocimientos. La traducción puede considerarse la base de una real sostenibilidad (inter)cultural, sólo si se aleja del individualismo y reconoce la diversidad (inaplazable reto de la era global), manteniendo siempre viva la convicción de que el lenguaje supone un equilibrio muy sutil entre las letras y sus silencios. Lo cual implica la necesidad del traductor de entrar en contacto con el residuo semántico de las palabras para revelar «il narrabile che nessuno racconta» $y$ «lo scrivibile che attende d'essere scritto» (Calvino 2002: 144). 
La traducción, como la memoria, vive constantemente entre conservación y remoción, muestra y al mismo oculta, crea realidades, pero también las destruye. Para impedir que la lengua erija barreras y para realizar una «hospitalidad lingüística» ética y responsable, el traductor tiene que abandonar cualquier mirada etnocéntrica, aceptando la idea de que no puede "vivir sólo en una lengua» y que traducir significa también someterse a ese mecanismo violento de perpetuas metamorfosis implícito en el viaje hacia el encuentro con una alteridad reconocida y, sobre todo, reconocible.

El acto traductivo es una experiencia hermenéutica de la alteridad. El traductor vive constantemente entre lo explícito y lo implícito de cada palabra, navega entre las dos orillas de un único río: la orilla de lo que se puede expresar y la orilla de lo que queda inexpresable. Los significados tácitos de la lengua (que son precisamente los que pueden caer en el olvido) ocultan la unicidad de una cultura con su historia y sus memorias. Preservar esa unicidad implica la capacidad de penetrar realmente en dicha cultura, huyendo de cualquier forma (consciente o inconsciente) de coerción para adoptar una perspectiva dialógico-relacional.

\section{BIBLIOGRAFÍA}

AA.W. 1984. Nunca Más. Informe de la comisión nacional sobre la desaparición de personas. Buenos Aires: Eudeba.

ALETTA DE SYLVAS, Graciela. 2011. «Memoria para armar». Acontracorriente, 8 (3): 140-162.

ALEXANDER, Jeffrey. 2004. «On the Social Construction of Moral Universals». Cultural Trauma and Collective Identity, ed. por J. C. Alexander, R. Eyrman, B. Giesen, N. Smelser y P. Szompka. Berkeley: University of California Press, 190-257.

ANGUIANO Peña y C. NAUMIS PEÑA. 2015. «Extracción de candidatos a términos de un corpus de la lengua general». Investigación Bibliotecológica, 29 (67): 19-45.

BABIČ, Željka. 2018. «A Re-reading of Transculturality in the Translation of Poetry». En La circolazione dei saperi in Occidente: teoria e prassi della traduzione letteraria, ed. por Fabio Scotto y Marina Bianchi. Milán: Cisalpino-collana Saggi CISAM, 109-135.

BACHTIN, Michail. 1992. The Dialogic Imagination: Four Essays. Austin: University of Texas Press.

BARTHES, Roland. 1983. El grano de la voz, [orig. Le grain de la voix] (Trad. Esp. de Nora Pasternac). Ciudad de México: Siglo XXI.

BASSNETT, Susan y Esperança BIELSA. 2009. Translation in Global News, London-New York: Routledge.

BAUMAN, Zygmunt. 2002. La cultura como praxis. [orig. Culture as Praxis] (Trad. Esp. Albert Roca). Barcelona: Paidós.

BERMAN, Antoine. 2003. La prueba de lo ajeno. [orig. L'épreuve de l'étranger] (Trad. Esp. de Rosario García López). Las Palmas: Universidad de Las Palmas de Gran Canaria.

CALVINO, Italo. 2002. Se una notte d'inverno un viaggiatore. Milán: Oscar Mondadori.

CRONIN, Michael. 2000. Across the Lines: Travel, Language, Translation. Cork: Cork University Press.

Rossella MICHIENZI

Traducir el blanco de las letras: semánticas de la memoria y sus evidencias cuali-cuantitativas 
FOUCAULT, Michel. 2002. Siete sentencias sobre el séptimo ángel (Con un ensayo de Ángel Gabilondo). [orig. Sept propos sur le septième] (Trad. Esp. de Isidro Herrera). Madrid: Arena libros.

GÁNDARA, Alejandro. 1998. Las primeras palabras de la creación. Barcelona: Anagrama.

GRIJELMO, Alex. 2000. La seducción de las palabras. Madrid: Taurus.

HALBWACHS, Maurice. 1935. Les cadres sociaux de la mémoire. París: Félix Alcan.

HANENBERG, Peter. 2018. «Intramental translation. How culture shapes the mind or why Columbus did not discover America». En La circolazione dei saperi in Occidente: teoria e prassi della traduzione letteraria, ed. por Fabio Scotto y Marina Bianchi. Milán: Cisalpino collana Saggi CISAM, 1-26.

KRISTEVA, Julia. 1991. Strangers to Ourselves, [orig. Étrangers à nous-mêmes] (Trad. Ing. de Louis S. Roudiez). Nueva York: Columbia University Press.

MESCHONNIC, Henri. 2011. Ethics and Politics of Translating. (Ed. y Trad. por Pier Pascale Boulanger). Ámsterdam-Filadelfia: John Benjamins.

MICHIENZI, Rossella. 2016. Racconti dell'Indicibile. Trauma e Memoria in Luisa Valenzuela. Milán: Rayuela Edizioni.

MICHIENZI, Rossella. 2015. La Traduzione del Trauma. Dallo studio dei linguaggi della memoria alla proposta di un modello traduttivo a partire dai racconti letterari e biografici sui desaparecidos argentini. Tesis doctoral. Università della Calabria.

RICOEUR, Paul. 2001. La traduzione. Una sfida etica. Ed. por Domenico Jervolino, traducido por Ilario Bertoletti y Mara Gasbarrone. Brescia: Editrice Morcelliana.

SALES SALVADOR, Dora. 2001. «Transculturación narrativa: posibilidades de un concepto latinoamericano para la teoría y la literatura comparada intercultural». Exemplaria. Revista de Literatura Comparada/Journal of Comparative Literature 5: 21-37.

SAMOJEDNY, Carlos. 1986. Psicología y dialéctica del represor y el reprimido. Experiencias de la unidad carcelaria 6-Rawson. Buenos Aires: Roblanco.

SELL, Jonathan P.A. 2018. "Metamorphosing the Human Text: Recognition in Literary Translation». En La circolazione dei saperi in Occidente: teoria e prassi della traduzione letteraria, ed. por Fabio Scotto y Marina Bianchi. Milán: Cisalpino - collana Saggi CISAM, 27-53.

SHAKESPEARE, William. 2005. The Complete Works. (Ed. por John Jowett, William Montgomery, Gary Taylor y Stanley Wells). Oxford: Clarendon Press.

Todorov, Tzvetan. 1995. Les abus de la mémoire. París: Arléa.

TRILLING, Lionel. 1971. Sincerity and Authenticity. Cambridge: Harvard University Press.

TYMocZKO, Maria y Edwin GENTZLER. 2002. Translation and Power. Amherst: University of Massachusetts Press.

VALENZUELA, Luisa. 2016. "Cambio de armas». En Racconti dell'Indicibile. Trauma e Memoria in Luisa Valenzuela, ed. por Rossella Michienzi. Milán: Rayuela Edizioni.

VIDAL CLARAMONTE, María Carmen África. 2017. "Dile que le he escrito un blues». Del texto como partitura a la partitura como traducción en la literatura latinoamericana. Madrid: Vervuert Iberoamericana.

VIDAL CLARAMONTE, María Carmen África y Rosario MARTín RuANO. 2013. Traducción, política(s), conflictos: legados y retos para la era del multiculturalismo. Granada: Editorial Comares.

Rossella MICHIENZI

Traducir el blanco de las letras: semánticas de la memoria y sus evidencias cuali-cuantitativas
CLINA

vol. 4-2, December 2018, 207-222

elSSN: 2444-1961

Ediciones Universidad de Salamanca - CC BY-NC-ND 